\title{
Projection-based Localization and Control Method of Robot Swarms for Swarm User Interfaces
}

\author{
TAKEFUMI HIRAKI*, The University of Tokyo, Japan \\ YOSHIHIRO KAWAHARA, The University of Tokyo, Japan \\ TAKESHI NAEMURA, The University of Tokyo, Japan
}

CCS Concepts: • Human-centered computing $\rightarrow$ Interaction devices; Ubiquitous and mobile computing systems and tools;

Additional Key Words and Phrases: pixel-level visible light communication, digital micromirror device, high-speed projector, augmented reality, human-computer interaction

ACM Reference Format:

Takefumi Hiraki, Yoshihiro Kawahara, and Takeshi Naemura. 2018. Projection-based Localization and Control Method of Robot Swarms for Swarm User Interfaces. Proc. ACM Interact. Mob. Wearable Ubiquitous Technol. 1, 1, Article 1 (October 2018), 6 pages. https://doi.org/10.1145/3267305.3267307

\section{INTRODUCTION AND SPECIFIC PROBLEMS}

The augmented reality systems, in which computer graphics and multiple mobile robots are cooperatively controlled, are useful for an intuitive understanding of complicated information because users can touch and operate information directly through robots $[10,18]$. The swarm user interfaces using cooperatively controlled multiple mobile robots are also a useful approach [11]. These systems express various modalities utilizing the assembly and distribution of robots; however, they cannot realize a collaboration between computer graphics and robots. Therefore, the system, in which computer graphics and multiple mobile robots seamlessly cooperate, can be expected to expand the domain of the swarm user interfaces.

Two problems remain in terms of ensuring a seamless collaboration between computer graphics and multiple mobile robots. First, previous methods use external measurement systems for localization that use the computer vision technology. However, the camera positions must be corrected and calibrated, and the spatial position of the robot in the camera images must be computed. Localization methods without computer vision may depend on a laser, sonar, or visible light communication [13]. However, these approaches have limited accuracy because of the resolution of each sensor. Augmented Coliseum [10] approaches this issue using a display-based measurement control system (DMCS) [19]. This technology eliminates the need for position-measuring devices and can support multiple robots on display. However, a prior initialization of tracking robots by marker-pattern images is necessary. Therefore, we cannot add or remove robots.

*This is the corresponding author

Authors' addresses: Takefumi Hiraki, The University of Tokyo, 7-3-1 Hongo, Bunkyo-ku, Tokyo, 113-8656, Japan, hiraki@nae-lab.org; Yoshihiro Kawahara, The University of Tokyo, 7-3-1 Hongo, Bunkyo-ku, Tokyo, 113-8656, Japan, kawahara@akg.t.u-tokyo.ac.jp; Takeshi Naemura, The University of Tokyo, 7-3-1 Hongo, Bunkyo-ku, Tokyo, 113-8656, Japan, naemura@nae-lab.org.

Permission to make digital or hard copies of all or part of this work for personal or classroom use is granted without fee provided that copies are not made or distributed for profit or commercial advantage and that copies bear this notice and the full citation on the first page. Copyrights for components of this work owned by others than ACM must be honored. Abstracting with credit is permitted. To copy otherwise, or republish, to post on servers or to redistribute to lists, requires prior specific permission and/or a fee. Request permissions from Permissions@acm.org.

UbiComp/ISWC'18 Adjunct, October 8-12, 2018, Singapore, Singapore

(C) 2018 Association for Computing Machinery.

ACM ISBN 978-1-4503-5966-5/18/10...\$15.00

https://doi.org/10.1145/3267305.3267307 
Second, the system load proportionally increases with the number of robots, which presents a scalability problem for controlling robots, because independent control signals via wireless or wired communication are often required in conventional methods. Other approaches, such as a simple direction control using multiple light sources [1], can navigate robots, but cannot guide them to an exact position. Thus, realizing a responsive control system for a large number of mobile robots without camera calibration while avoiding the system load problem is not trivial.

We have been studying the pixel-level visible light communication (PVLC) [9] by implementing the visible light communication technology with a high-speed projector. We can embed imperceptible information in each pixel of projected images using PVLC. By controlling multiple mobile robots using PVLC, we can solve the abovementioned three problems and realize the swarm user interfaces with the collaborative control of graphics and multiple robots. However, the image expression (color gradation, contrast) was poor, and the spatial resolution of the embedded information was low in the previous PVLC systems. The projector device for PVLC was large, and its portability was low. Furthermore, we needed to develop an information embedding method suitable for this to realize robust robot control against disturbance, such as displacement of robots by the users' touch.

\section{RELATED WORK}

We briefly reviewed herein robot localization methods, robot control methods, and methods that combined both localization and control using projected light patterns for communication. We also reviewed the PVLC technique utilized in the proposed method.

Localization methods using projected structured light and photosensors are conventional in the context of user interfaces and motion capture systems. RFIG Lamps [17] and Lee et al. [12] localized an object with photosensors using striped pattern Gray-code images. Prakash [16] utilized a unique projection device with transparent glass slides of Gray-code patterns and multiple LEDs that can flicker at a very high frequency. In all these methods, a receiver can estimate its two-dimensional position from spatial light patterns; however, directly adding more information, such as control commands for robots or images other than signal patterns, which are meaningless to the human eyes, is not trivial.

Robot control is usually performed after the robot is localized, but various methods use projected light for direct path guidance without localization. Fujiwara et al. [3] interactively controlled line-follower robots by letting users draw lines with hand gestures. Line-follower robots do not know their location, but instead run along a path that is visually defined. Hara et al. [4] proposed the dynamic path control of a single robot using a narrow laser beam. VisiCon [8] proposed a robot manipulation method using a hand-held projector. However, these approaches are not suitable for multiple robots.

Several methods implement both the localization and control of multiple mobile robots. Liu et al. [13] performed this task using visible light communication (VLC). Their system sends position information using general data communication, and feedback to the central system is not required. However, the localization accuracy is not very high because the position is estimated from the incident angle of the light.

Meanwhile, DMCS [19] achieves both localization and control. It consists of a conventional display device and multiple mobile robots with several photosensors evenly spaced on a robot's surface. DMCS does not require position measurement devices, such as color or depth cameras, and can support an unlimited number of robots on display. This initialization is necessary whenever a robot is added to the projection area, and it takes several seconds for each robot; thus, adding or removing robots is not possible.

We utilize PVLC to achieve an initialization-free and marker-free method that both localizes and controls an unlimited number of robots [9]. 
PVLC is a method that superimposes data patterns on pixels with human-imperceptible flicker using a very high-speed digital light processing (DLP) projector. PVLC can display a visual image containing superimposed data as bit patterns that are decodable by receiver circuits. When two inverted patterns are alternately displayed at a high frequency, the human eyes only see a flat gray image because of the persistence of a vision effect. Although the human eyes cannot distinguish each image, a receiver with a photosensor can detect the images as different signals. The embedding algorithm for determining the on and off periods must be carefully designed to maintain luminance and avoid flicker.

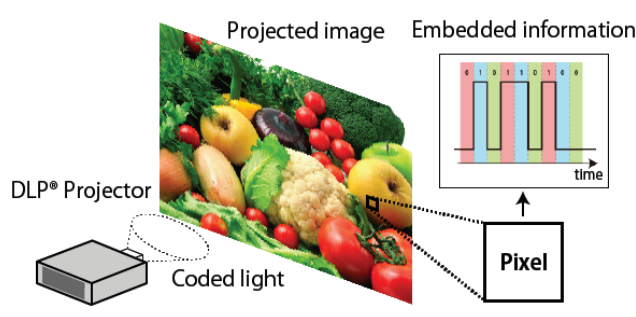

Fig. 1. Principle of PVLC. A PVLC system can superimpose data patterns on pixels using humanimperceptible high-speed flicker.

\section{METHODOLOGICAL APPROACHES AND THE KEY IDEAS}

We propose herein the projection-based localization and control method of multiple mobile robots using PVLC to solve the problems mentioned in the previous section. In our proposed robot control method, the positional displacement between projected images and robots does not occur in principle because robots use projected coordinates of a projector to control themselves (Figure 2). We realize the robot control method, which is robust against disturbance, and can freely add and remove robots by superimposing the information of velocity vector fields on the projected images. This feature can be realized because robots can acquire the projected position and control information with the same cycle as the control cycle of robots. Furthermore, we can intuitively manipulate multiple robots by moving the coordinate system itself to be controlled. Our proposed method has two main characteristics: swarm control concept and real-time controllability.

\subsection{Swarm Control Concept}

We explain the position of our proposed method in robot swarm control. Two kinds of control methods can be used for multiple mobile robots, and these are roughly divided into centralized control and distributed control.

In the centralized control system, a central computer measures the state of robots and controls them individually by communicating with each robot. With this system, we can individually and precisely control each robot by sending individual instructions to each robot. However, the scalability problem occurs as the number of robots increases, in which the communication load of the system proportionally increases.

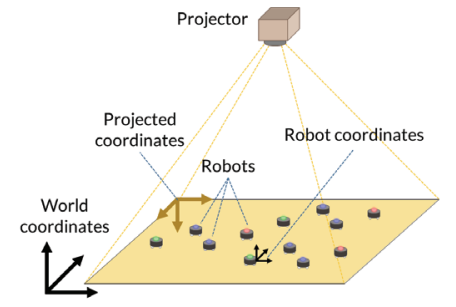

Fig. 2. Coordinates of the projection-based robot control system.

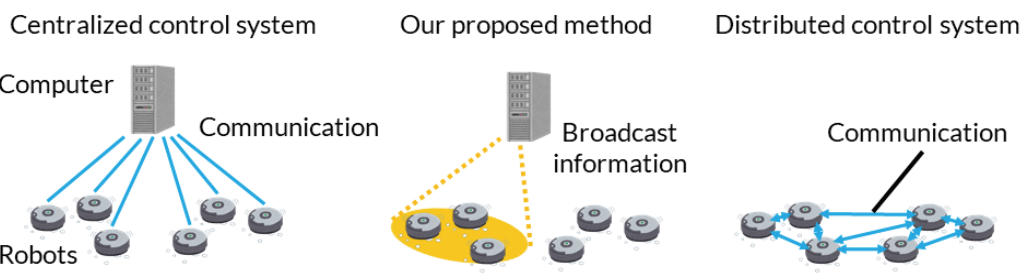

Fig. 3. Concepts of control methods for a swarm of robots. Our proposed method has the characteristics of both centralized control and distributed control. 
In the distributed control system, each robot communicates or senses each other to measure an environment and control itself. The scalability problem does not occur in this system because the problem of the communication load does not occur in principle. However, sending systematic control commands to each robot is impossible because no central computer is used.

Our proposed method can be regarded as a method combining features of both centralized control and distributed control. The information corresponding to a position is embedded in the projected images in this method, and the system broadcasts it to each robot. Our method cannot send control instructions for each robot unlike in centralized control; however, it can realize control as a swarm by sending the instructions according to a position. The scalability problem does not occur because the communication load of light communication is irrelevant to the number of robots. However, it cannot be controlled unless robots can physically fit within a projected image. Figure 3 shows the concept of these three control methods.

\subsection{Real-time controllability}

The significant difference between our proposed method and conventional projection-based robot control is the real-time controllability. In the field of automatic control, the real-time performance can be classified into four types: non-RT, soft-RT, hard-RT, and isochronous-RT $[2,15]$. Isochronous-RT has a control cycle of $1 \mathrm{~ms}$ or less and is used for precise motion control. Hard-RT has a control cycle of 1 to $10 \mathrm{~ms}$ and is used for general control. Soft-RT has a scalable control cycle of approximately $100 \mathrm{~ms}$ and is used in a factory floor and process automation. Non-RT has a control cycle exceeding $100 \mathrm{~ms}$.

In the computer vision-based localization method used in conventional systems, measurement takes several tens of ms, and data transmission by wireless communication takes more than $20 \mathrm{~ms}$ [14]. This method can be regarded as insufficient for robot con-

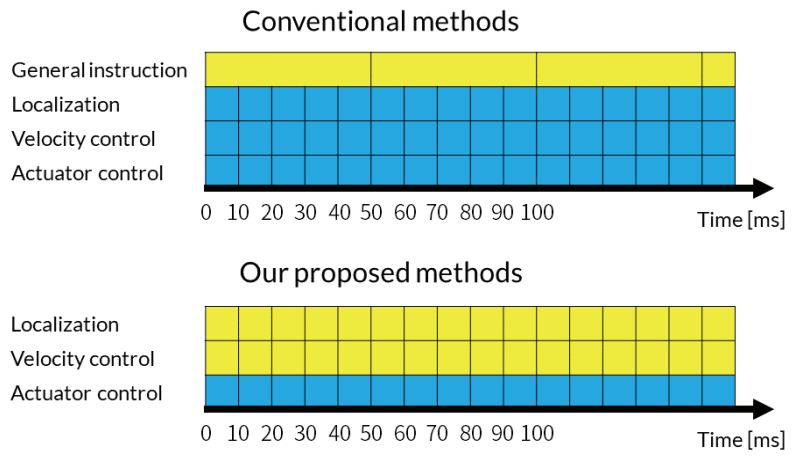

Fig. 4. Comparison of the processing time charts of the microcomputers between the conventional methods and our proposed methods from the viewpoint of real-time control. The processes indicated in yellow are updated by external communication, while those in light blue are executed by microcomputers.

trol because this process corresponds to soft-RT. In our proposed method, the update of information for localization and control is completed within $10 \mathrm{~ms}$ because the information is transmitted by light communication. As a result, the microcomputers of robots can use the received information in each cycle of the hard-RT control.

Figure 4 shows a comparison of the processing time charts of the microcomputers between the conventional methods and our proposed method. In the conventional method, general instructions, such as target points, are not updated for several control cycles. Moreover, robots are controlled based on these instructions and the localization performed by themselves. Meanwhile, in our proposed method, the information for localization and control is transmitted from the central system, and the job of the microcomputers is only to control the robot actuators based on this information. Consequently, sensors, such as rotary encoders and gyro sensors, for position measurement become unnecessary for robots, and we can inexpensively, compactly, and efficiently design robots.

\section{RESEARCH CONDUCTED AND PLANNED AHEAD}

We conducted the following three projects to enhance the interactivity of the swarm robot interfaces by realizing robot localization and control method using PVLC: (1) Fundamental development of PVLC for improvement 
of the image expression and the spatial resolution of information. (2) Phygital Field: a localization and control system of multiple mobile robots to realize direct touch operation of robots. (3) NavigaTorch: an interface system that operates multiple mobile robots by illuminating robots with the projected images of a handheld PVLC system.

\subsection{Fundamental Development of Pixel-level Visible Light Communication}

We designed and implemented a new PVLC projector capable of high-speed data transfer to improve the image expression and the spatial resolution of embedded information [7]. The key idea of this research is the use of the difference between the maximum blinking frequency of digital micromirror devices (DMD), which is a pixel control element of a projector and light-source LEDs. We can efficiently transmit data by blinking LEDs in synchronization with the control of DMD. Our proposed system can be used on general PCs because the PVLC data are transferred from the PC to the projector using the HDMI interface. We designed and implemented a light-receiving sensor circuit compatible with this high-speed blinking light. This circuit can separate ambient disturbance light and signal light using a high-pass filter circuit. In evaluation experiments, we clarified the trade-off and performance limit of our proposed system, that is, the optimum blinking frequency is $48 \mathrm{kHz}$ for the light source, and it is $3 \mathrm{kHz}$ for the DMD.

\subsection{Phygital Field}

We proposed Phygital Field, a localization and control system of multiple mobile robots for realizing direct touch operation [5,6]. Robots must be robustly controlled against the interference of PVLC information and disturbance caused by users to realize the interaction in which bodies of users directly touch robots. We propose a stable localization method by embedding the position coordinate information considering interference and a movement control method by embedding velocity vector field information. We also demonstrate these methods by developing a new PVLC projector and small robot swarms. In the evaluation experiments, we have confirmed that robots can localize their positions with errors within a few $\mathrm{mm}$ from ground truth, and that the target point tracking control of robots is possible by updating the velocity vector fields.

\subsection{NavigaTorch}

We proposed NavigaTorch, a robot operation interface by illuminating robots with the light of our developed projector. We built a portable PVLC system using a compact and lightweight DLP projector. Projecting the PVLC video using the previous PVLC method was impossible because the built-in memory capacity of this projector was small. We implemented a PVLC system using this projector by implementing the new encoding algorithm of the PVLC data. Specifically, we successfully reduced the amount of transfer data by decoding the image part of the PVLC data as normal image data instead of binary data and projecting it as it is. As a result, the image represented by $N$ binary images in the previous method can be represented by $\log _{2}(N)$ images. In the evaluation, we projected a PVLC video at a refresh rate of $60 \mathrm{~Hz}$ and confirmed that robots could be controlled and operated.

\section{CONTRIBUTION IN THE FIELD OF UBIQUITOUS COMPUTING}

The expected contribution of this research is to propose a method, which realizes a collaborative control of computer graphics and robots, and implement applications using the method. Specifically, we realize the localization and control method using PVLC, which has both the merits of a centralized control system and a distributed control system, and the real-time controllability matching the control cycle of robots. When we regard our proposed system as a lighting device instead of a video-projecting device, it can be expected to be applied to various fields requiring indoor localization. 


\section{ACKNOWLEDGMENTS}

This work was supported by JST ERATO Grant Number JPMJER1501, JSPS KAKENHI Grant Number JP16H01739, and Grant-in-Aid for JSPS Fellows JP17J04216, Japan.

\section{REFERENCES}

[1] Aaron Becker, Golnaz Habibi, Justin Werfel, Michael Rubenstein, and James McLurkin. 2013. Massive uniform manipulation: Controlling large populations of simple robots with a common input signal. In Proceedings of 2013 IEEE/RSf International Conference on Intelligent Robots and Systems. 520-527. https://doi.org/10.1109/IROS.2013.6696401

[2] Peter Danielis, Jan Skodzik, Vlado Altmann, Eike Bjoern Schweissguth, Frank Golatowski, Dirk Timmermann, and Joerg Schacht. 2014. Survey on real-time communication via ethernet in industrial automation environments. In Proceedings of the 2014 IEEE Emerging Technology and Factory Automation (ETFA). 1-8.

[3] Taiki Fujiwara and Yasushi Iwatani. 2011. Interactions with a line-follower: An interactive tabletop system with a markerless gesture interface for robot control. In Proceedings of 2011 IEEE International Conference on Robotics and Biomimetics. 2037-2042.

[4] Keigo Hara and Shoichi Maeyama. 2008. Navigation Using One Laser Source for Mobile Robot with Optical Sensor Array Installed in Pan and Tilt Mechanism. In Proceedings of 2008 IEEE/ASME International Conference on Advanced Intelligent Mechatronics. 257-262.

[5] Takefumi Hiraki, Shogo Fukushima, and Takeshi Naemura. 2016. Phygital Field: an Integrated Field with a Swarm of Physical Robots and Digital Images. In SIGGRAPH ASIA 2016 Emerging Technologies on - SA '16. Article No.2.

[6] Takefumi Hiraki, Shogo Fukushima, and Takeshi Naemura. 2016. Projection-based Localization and Navigation Method for Multiple Mobile Robots with Pixel-level Visible Light Communication. In 2016 IEEE/SICE International Symposium on System Integration (SII). $862-868$.

[7] Takefumi Hiraki, Mika Koizumi, Leijie Zhou, Shogo Fukushima, and Takeshi Naemura. 2016. Reconfigurable Pixel-level Visible Light Communication with Light Source Control. Transaction on the Virtual Reality Society of fapan 21, 1 (2016), 197-206.

[8] Kazuhiro Hosoi, Vinh Ninh Dao, Akihiro Mori, and Masanori Sugimoto. 2007. VisiCon: A Robot Control Interface for Visualizing Manipulation Using a Handheld Projector. In Proceedings of the international conference on Advances in computer entertainment technology - ACE '07. 99-106.

[9] Sho Kimura, Ryo Oguchi, Hideo Tanida, Yasuaki Kakehi, Keita Takahashi, and Takeshi Naemura. 2008. PVLC Projector: Image Projection with Imperceptible Pixel-Level Metadata. In ACM SIGGRAPH 2008 Posters on - SIGGRAPH '08. Article No.135.

[10] Minoru Kojima, Maki Sugimoto, Akihiro Nakamura, Masahiro Tomita, Masahiko Inami, and Hideaki Nii. 2006. Augmented Coliseum: An Augmented Game Environment with Small Vehicles. In Proceedings of First IEEE International Workshop on Horizontal Interactive Human-Computer Systems - TABLETOP '06. 3-8.

[11] Mathieu Le Goc, Lawrence H. Kim, Ali Parsaei, Jean-Daniel Fekete, Pierre Dragicevic, and Sean Follmer. 2016. Zooids: Building Blocks for Swarm User Interfaces. In Proceedings of the 29th Annual Symposium on User Interface Software and Technology - UIST '16. 97-109.

[12] Johnny C. Lee, Paul H. Dietz, Dan Maynes-Aminzade, Ramesh Raskar, and Scott E. Hudson. 2004. Automatic Projector Calibration with Embedded Light Sensors. In Proceedings of the 17th annual ACM symposium on User interface software and technology - UIST '04. $123-126$.

[13] Xiaohan Liu, Hideo Makino, and Kenichi Mase. 2010. Improved Indoor Location Estimation Using Fluorescent Light Communication System with a Nine-Channel Receiver. IEICE Transactions on Communications E93-B, 11 (nov 2010), 2936-2944.

[14] Vladimir Lucan, Petr Simacek, Jari Seppälä, and Hannu Koivisto. 2003. Bluetooth and Wireless LAN Applicability for Real-Time Control. In Proceedins of Automaatio 2003. 35-40.

[15] Peter Neumann. 2007. Communication in industrial automation-What is going on? Control Engineering Practice 15, 11 (nov 2007), $1332-1347$.

[16] Ramesh Raskar, John Barnwell, Shree Nayar, Masahiko Inami, Philippe Bekaert, Michael Noland, Vlad Branzoi, Erich Bruns, Hideaki Nii, Bert DeDecker, Yuki Hashimoto, Jay Summet, Dylan Moore, Yong Zhao, Jonathan Westhues, and Paul Dietz. 2007. Prakash: Lighting Aware Motion Capture using Photosensing Markers and Multiplexed Illuminators. ACM Transactions on Graphics 26, 3 (jul 2007), 36.1-36.11.

[17] Ramesh Raskar, Paul Beardsley, Jeroen van Baar, Yao Wang, Paul Dietz, Johnny Lee, Darren Leigh, and Thomas Willwacher. 2004. RFIG Lamps : Interacting with a Self-Describing World via Photosensing Wireless Tags and Projectors. ACM Transactions on Graphics 23, 3 (aug 2004), 406-415.

[18] D. Rosenfeld, M. Zawadzki, J. Sudol, and K. Perlin. 2004. Physical Objects as Bidirectional User Interface Elements. IEEE Computer Graphics and Applications 24, 1 (jan 2004), 44-49.

[19] Maki Sugimoto, Kazuki Kodama, Akihiro Nakamura, Minoru Kojima, and Masahiko Inami. 2007. A Display-Based Tracking System: Display-Based Computing for Measurement Systems. In Proceedings of 17th International Conference on Artificial Reality and Telexistence (ICAT 2007). 31-38. 